\title{
Investigation of Cascading Events in Power Systems with Renewable Generation
}

\author{
Georgios A. Nakas, Student member, IEEE, Panagiotis N. Papadopoulos, Member, IEEE \\ Department of Electronic and Electrical Engineering \\ University of Strathclyde \\ Glasgow, UK \\ georgios.nakas@strath.ac.uk,panagiotis.papadopoulos@strath.ac.uk
}

\begin{abstract}
The paper introduces a framework for characterisation and investigation of cascading events in power systems with renewable generation using time domain dynamic simulations. The paper aims at identifying the cascading event patterns by including protection device operation in RMS simulations and analyzing them. The cascading events are characterised by the power system components involved, the sequence of trippings and the reason for failure (e.g. voltage/frequency), while considering a wide range of possible operating conditions defined by economic dispatch. Changes in observed cascading failure patterns for different operating conditions are identified and investigated, taking also into consideration the impact of renewable generation. The framework is demonstrated on a modified version of the Anderson-Fouad 9 bus model incorporating renewable generation and protection devices.
\end{abstract}

Keywords-cascading events, cascading failures, dynamic simulation, protection, renewable generation

\section{INTRODUCTION}

Better understanding the nature of cascading events and the dynamics involved is of significant importance in securing against and preventing blackouts. So far, even in power systems dominated by synchronous generation, cascading events have not been very well understood. In recent years, the penetration of renewable energy sources (RES) as well as other power electronic interfaced devices (e.g. HVDC interconnectors) is drastically increasing in most countries worldwide, causing increased uncertainty in power system operation and significant changes in power system dynamic behaviour. This wider variation in power system operating conditions and different dynamic behaviour can potentially lead to unforeseen dynamic interactions which might lead to cascading events and in the worst case, blackouts.

Identifying and investigating cascading failures is a very challenging task, since dynamics at various timescales as well as actions of protection devices need to be properly represented. In addition, a large number of possible initial operating conditions (including different system loading and generation output as dictated by economic dispatch) and contingencies need to be accounted for [1], [2].

In the literature, there are several methods investigating cascading failures using static calculations as a tool. The method proposed in [3] uses a load flow computation for evaluating and creating risk-based indices of the contingencies. Using pattern recognition and fuzzy estimation, the most probable failure sequences are identified online for various operating conditions.

In [4] a method based on a stochastic "Random Chemistry" algorithm using DC power flow is proposed in order to identify sets of multiple contingencies that cause large cascading failures. Starting from a large set of contingencies that cause cascading failures, subsets of the original set are randomly picked up and simulated until a smaller subset that causes cascading failures is found. This subset then takes the place of the original set and the same procedure is repeated until the minimum number of contingencies that causes cascading failures is found. A different approach using the same simulator and case data as in [4] is presented in [5], in which the results from many cascading simulations are used to create an influence graph that provides information on how the cascades evolve in a particular system. It is also noted that the cascading events do follow patterns, the identification of which is useful in reducing the risk of large cascading blackouts.

However, it has been shown that dynamic simulations provide more details and accuracy than static, at the expense of larger computational effort. A comparison between static and time domain simulations running on the same test system and applying the same contingencies is presented in [6]. The results reveal a good consistency in the early steps of the simulation, however the last evolutions are not accurately caught by the static model because of the occurrence of fast dynamic phenomena.

A method for identifying cascading events using time domain dynamic simulations is presented in [7]. A search algorithm for identifying plausible harmful N-k contingencies is demonstrated on a network based only on synchronous generation. Dynamic time-domain simulations were used to assess cascading events sequences that cause instability or non-viable system conditions, after applying initial contingencies.

In this paper a framework for the characterisation of cascading events in power systems with renewable generation is developed, based on long duration RMS time domain dynamic simulations. The main contribution of the paper is the investigation of the impact that RES have on the appearance of cascades, by considering a wide range of initial operating conditions for different system loading and intermittent renewable generation. The investigation starts from an optimal power flow (OPF) solution to capture the effect of initial steady-state conditions. A novel aspect of the paper is to also include discrete protection device operation that can capture in detail device tripping related to various aspects of transient, voltage and frequency stability. The combination of longer duration time domain simulations and inclusion of protection device operation introduces a way of analysing the cascading event patterns in detail, based on the frequency of appearance, the power system components involved, the reasons for tripping (e.g. voltage or frequency) and how these change for different operating conditions. This information could be vital in exposing network vulnerabilities and designing preventive as well as corrective measures to avoid cascading events from spreading.

This work was funded by EPSRC. All results can be fully reproduced using the methods and data described in this paper and provided references. 


\section{MethodOLOGY}

\section{A. Detailed procedure}

In order to perform a comprehensive cascading events study, there is need for detailed representation of related fast and slow dynamics, protection device operation as well as prefault operating conditions affected by cost of generation (in operation driven by economic dispatch), variation of system load and intermittent renewable generation [1]. In this study, a dynamic RMS model of a test network with high penetration of RES, including protection devices is used to identify and characterise possible cascading events. The model represents dynamic phenomena related to voltage, frequency and transient stability and includes the actions of protection devices related to over-/under- voltage and frequency, pole slip protection and distance protection. In addition, a basic load shedding scheme is modelled, normally present in power system operation as a defence mechanism to arrest significant frequency drops after loss of generation. Automatic voltage regulators, over-excitation limiters, power system stabilizers, , wind generator detailed controllers, tap changer actions and governors are also modeled to capture slower voltage related phenomena as well as primary frequency response actions within a timeframe of 3 minutes. The goal is to capture any instability phenomena that occur within this timeframe and also discrete actions of protection devices that can significantly affect the trajectory of system states.

A brute force approach is followed to simulate a wide range of possible operating conditions. This is performed by discretising multiple variables such as system loading and wind penetration, as described later, that also include stressed network situations. After considering the initial operating conditions for load and wind generation, an Optimal Power Flow (OPF) problem is solved in order to determine the dispatch of conventional generators.

Dynamic RMS simulations are performed to capture the system response to an applied contingency. Three phase faults on lines are considered as initiating events in this study. The faults get cleared by the protection devices included in the model, and in some cases lead to a cascading event sequence involving multiple failures. The cascading events are caused by tripping of components, due to intentional interventions of the protection devices after the relevant limits are violated (e.g. under-/over- voltage or frequency). The cascading events are then characterised by the component that is disconnected (capturing locational aspects), the time of disconnection (capturing the sequence of events) and the reason for disconnection (capturing the potential instability mechanism).

For the possible discretized operating conditions and contingencies that are considered, the patterns in which the cascading events occur are identified. These patterns are characterised by metrics relative to frequency of appearance, whether they lead to a viable or non-viable case, the reason of the first cascading event, the specific components that trip along with the reason for tripping, and how these metrics relate to the amount of wind penetration and system loading.

The aim of this characterisation and introduction of above mentioned metrics is to identify vulnerabilities of the network, related to instability mechanisms and areas or specific components that might lead to onset of cascades. In addition, describing a potential sequence of cascades in detail can offer insights into strengthening the network to prevent cascades from occurring or designing special protection schemes to stop them at their onset.

\section{B. System under Study}

In this study, a modified version of the Anderson-Fouad 9 bus model (Fig. 1.) [8] is used. The system is implemented using RMS simulations in DIgSILENT PowerFactory [9]. The network nominal voltage is $230 \mathrm{kV}$, and the nominal frequency is $60 \mathrm{~Hz}$.

The three synchronous generators (SGs) in the network are represented by full detail four winding models (6th-order). $\mathrm{SG} 1$, the reference machine of the system, is a hydro type machine equipped with a governor (GOV). SG2 and SG3 are coal type generators equipped with IEEE DC1C Automatic Voltage Regulator (AVR), Power System Stabilizer (PSS), Governor (GOV), and Over-excitation Limiter (OEL). All generators have an operating region from 0.30 to 1 p.u. active power loading. More details about the system parameters can be found in [10].

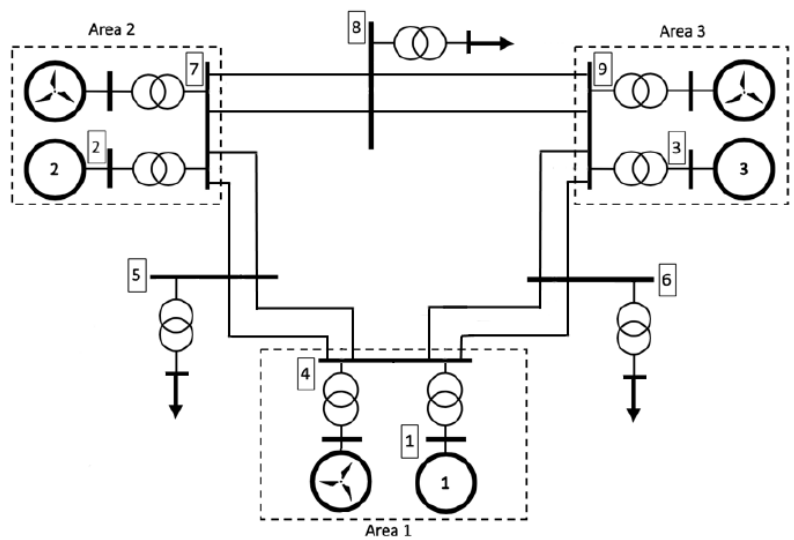

Fig. 1. Modified version of the Anderson-Fouad 9 bus model.

The wind generators in this study are modeled using International Electrotechnical Commission (IEC) type 4A wind turbines [11]. The installed capacity of wind generation is considered to be equal to $20 \%$ of the installed conventional generation of each area, which is 247.5MVA, 192.0MVA and 128.0MVA for area 1, 2 and 3 respectively. The entire range of possible operating conditions for each wind farm is considered, as described in more detail in Section III.A.

In this paper the focus is on developing the general approach to investigate cascading failures including the modelling of protection devices, in systems with renewable generation. For this reason, a small but widely used model is used as a benchmark. This also allows us to practically simulate a very high number of cases (154026) to capture most possible operating conditions as the focus of this paper is not to develop an efficient method of identifying cascading failures.

Future work focuses on the application of a search algorithm for the investigation of cascading events which will allow the use of more realistic larger networks (e.g. the IEEE39 bus test system.).

\section{Discretizing System Loading and Wind Penetration Values}

The system loading and wind generation output values are discretised with a certain step between a minimum and maximum allowable range as described below. The maximum wind penetration amount per case is defined by the system 
loading, the operating range of the synchronous generators and the nominal capacity of the installed wind generation. The minimum active power dispatch of synchronous generators and the system loading set the limit for the maximum possible wind generation as the wind and synchronous generation output must equal the system loading and the network power losses. The values used in this study are described in Section III.A

\section{AC OPF and Conventional SG Disconnection}

An AC OPF [12] is solved to determine the dispatch of the SGs using the inbuilt OPF solver function in DIgSILENT Powerfactory. Each SG is allocated either a high, medium or low cost, as in [8], establishing a merit order among the SGs. The objective of the OPF is the minimisation of the total synchronous generation cost, while respecting constraints set by the active and reactive power limits of the generators, the maximum loading of the lines and the bus voltage limits. $\mathrm{SG}_{1}$ is allocated a medium, $\mathrm{SG}_{2}$ a low and $\mathrm{SG}_{3}$ a high incremental cost.

The amount of conventional SG disconnection, and consequent inertia variation in the network, as the wind and load varies, is also considered in a simple manner after the OPF solution. Each generator is assumed to have $15 \%$ additional spare capacity (headroom) on top of the operating point taken from the OPF solution, assuming this does not violate its initial nominal capacity [13]. If the resulting nominal capacity is larger than the initial nominal power of the generator (as described in [10]), then it is set to the initial nominal value. In this case, there is no room for further disconnection of conventional generation.

\section{E. Load Tap Changer}

The loads are connected to distribution rating voltage buses and are modelled as balanced three-phase constant impedance loads. The loads are connected to the network via step-down transformers, equipped with Load Tap Changers (LTCs). The LTCs adjust the transformer ratios keeping the distribution voltage within the deadband [0.99-1.01] p.u. When the distribution voltage leaves this deadband, the LTC acts after an intentional delay of 10s. The LTCs adjust the transformer ratios in the range [-6.5\% to $+6.5 \%]$ over 8 positions. For each step the ratio varies by $1.63 \%$.

\section{F. Protection Devices}

The following protection devices have been modelled in the network:

1) SGs protection: The generators are equipped with an under-/over-speed protection relay $[-6 \% /+4 \%]$, an undervoltage protection relay (ranging from 0 to 0.9 p.u. including multiple steps with different delays), and pole-slip protection.

2) RES protection: The non-synchronous generators (NSGs) in the system are protected with an under-/overvoltage protection relay with FRT (ranging from 0.15 p.u. to 1.2 p.u. including various steps with different delays) and an under-/over-frequency protection relay $[-6 \% /+4 \%]$.

3) An Under-Frequency Load Shedding (UFLS) scheme with four stages was implemented for the disconnection of a percentage of demand at low frequency. (from $-3 \%$ to $-4 \%$ of the nominal frequency, with each stage disconnecting $10 \%$ of demand)

4) Transmission line protection: Each line is protected by two distance protection relays positioned at the line ends. The relays have two zones of protection: the first one is set at $80 \%$ of the line's reach and acts instanteneously and the second one is set at $120 \%$ of the line's reach with a $400 \mathrm{~ms}$ delay. An inter-tripping scheme between the relays has also been modelled.

All the protection devices were implemented using standard models found in the DIgSILENT Powerfactory library. The relays settings have been adopted from the UK grid code to comply with the settings for protection devices connected in the transmission system as referred in [14].

\section{RESULTS}

\section{A. Test Cases}

In modern power systems operation there is a large number of uncertainties to be considered. It is therefore important to take into consideration a large number of possible cases to investigate the system response to a contigency. In this paper, a brute force approach is followed, by sampling almost every plausible pre-fault system operating condition, including stressed network conditions and applying faults to different locations of all lines in the system as initiating events. The effect of the fault location, the system loading and the amount of wind penetration is investigated. The system loading and wind generation output values are discretised between a minimum and maximum allowable range as described in Section II.C with resulting values shown below. The SG and RES network parameters are shown in Table I.

The system loading is assumed to range from $60 \%$ to $130 \%$ of the total network demand (315MW, 115MVar) as described in [9], in $10 \%$ steps. The output of each of the three wind generators in the network ranges from 0 to the maximum allowable in $10 \%$ steps. It should be noted that the wind generation output $\%$ value in all results refers to the assumed wind installed capacity as described in Section II.B. e.g. $100 \%$ maximum total wind generation output means $100 \%$ out of the installed wind capacity. In every case there is an amount of synchronous generation in the network, as defined by the AC OPF solution. In this study 154026 cases in total were simulated. Three phase faults in three different locations $(10 \%, 50 \%, 90 \%)$ on each Line (1-6) are considered as initiating events. That gives 18 different cases for each given network operating condition, multiplied by 8 different loading scenarios and by the allowable RES output scenarios.

TABLE I. SG AND RES NETWORK PARAMETERS

\begin{tabular}{|c|c|c|}
\hline \multicolumn{3}{|c|}{ SG Machine Rating } \\
\hline $\begin{array}{c}\text { SG1 } \\
M V A \\
\end{array}$ & $\begin{array}{c}S G 2 \\
M V A\end{array}$ & $\begin{array}{c}\text { SG3 } \\
\text { MVA } \\
\end{array}$ \\
\hline 247.5 & 192.0 & 128.0 \\
\hline \multicolumn{3}{|c|}{$\begin{array}{c}\text { Minimum Active Power Dispatch } \\
\text { (30\% of Machine Rating) }\end{array}$} \\
\hline $\begin{array}{l}S G 1 \\
M W\end{array}$ & $\begin{array}{l}S G 2 \\
M W\end{array}$ & $\begin{array}{l}S G 3 \\
M W\end{array}$ \\
\hline 74.25 & 57.6 & 38.4 \\
\hline \multicolumn{3}{|c|}{ Wind Generation Installed Capacity } \\
\hline $\begin{array}{c}\text { NSG1 } \\
M W\end{array}$ & $\begin{array}{c}N S G 2 \\
M W\end{array}$ & $\begin{array}{c}\text { NSG3 } \\
M W\end{array}$ \\
\hline 49.5 & 38.4 & 25.6 \\
\hline
\end{tabular}

The duration of the RMS simulations has been set to 180 $\mathrm{s}$. The simulations have been performed on a standard desktop computer (CPU Intel i7-8700 3.20 GHz, 16 GB RAM) using the interface between Python and DIgSILENT Powerfactory [15]. 
The approximate time that a single case simulation run takes is 6s. A parallel processing approach has been implemented to speed up the process, by running multiple (up to four in this paper) simulated cases in parallel. It should be noted that due to the large computational effort this brute force approach might be challenging for real scale large networks. While this approach refers to planning phase where more time is available for studies, still an importance or efficient sampling technique might need to be adopted [16], [17] or a screening technique as in [7].

\section{B. Cascading Events Characterisation}

In this study, cascading events appeared in 31250 cases, out of the 154026 that are simulated $(20.3 \%$ of the simulated cases), $15031(9.8 \%)$ of which led to system collapse. These cascading events appeared in 161 different patterns. As shown in Table II, all of the cases with cascading events occurred for increased system loading, at 110, 120 and $130 \%$. When the system loading increases from 110 to $120 \%, 67$ out of the 84 patterns that appear are new, and when the loading increases from 120 to $130 \% 69$ out of the 143 patterns are new. Higher system loading leads to more frequent appearance of cascading events and an increase in the number of different patterns, as the network operates closer to its limits.

TABLE II. NUMBER OF CASES PER PARAMETER

\begin{tabular}{|c|c|c|c|}
\hline $\begin{array}{c}\text { System } \\
\text { Loading } \\
(\boldsymbol{\%})\end{array}$ & $\begin{array}{c}\text { Number of Cases } \\
\text { with Cascading } \\
\text { Events }\end{array}$ & $\begin{array}{c}\text { Number of Cases } \\
\text { leading to System } \\
\text { Collapse }\end{array}$ & $\begin{array}{c}\text { Number of } \\
\text { Cascading } \\
\text { Event Patterns }\end{array}$ \\
\hline Up to 100 & 0 & 0 & 0 \\
\hline 110 & 1313 & 479 & 20 \\
\hline 120 & 12066 & 4909 & 84 \\
\hline 130 & 17871 & 9643 & 143 \\
\hline \multirow{2}{*}{} & $\begin{array}{c}\text { Total Number of } \\
\text { Cases with } \\
\text { Cascading } \\
\text { Events }\end{array}$ & $\begin{array}{c}\text { Total Number of } \\
\text { Cases leading to } \\
\text { System Collapse }\end{array}$ & $\begin{array}{c}\text { Total Number } \\
\text { of Cascading } \\
\text { Event Patterns }\end{array}$ \\
\cline { 2 - 4 } & 31250 & 15031 & 161 \\
\hline
\end{tabular}

1) Most common cascading events patterns: In Table III the most common cascading events patterns that have appeared are presented. The component name is followed by the reason of disconnection, e.g. G2-UV means that the component $\mathrm{G} 2$ disconnected by the under-voltage protection relay. The three most common patterns involve the disconnection of RES units only due to under-voltage and do not cause any further trips of other components. The rest of the patterns, involve the disconnection of synchronous generation due to under-voltage, that leads to a drop in the frequency of the system, which causes the disconnection of loads due to under-frequency and eventual disconnection of all the syncronous machines. Out of the 161 total patterns, 156 of them result in non-viable cases. It should be noted that in these 156 patterns G2 is the first SG that gets disconnected. This is the machine with the lowest cost, and as a result from the solution of OPF, has the highest loading.

To highlight how the characterisation of cascading events in the suggested way can be helpful, a suggestion for this specific system is to improve voltage support close to wind farms located mainly in area 2 . This can be done either in planning timescale by potentially adding devices (e.g. FACTS) or in operational timescales by ensuring reactive support from nearby generators is available. Consequently, this could help stop more serious cascading events from evolving that can lead to disconnection of loads.

TABLE III. MOST COMMON CASCADING EVENTS PATTERNS

\begin{tabular}{|c|c|}
\hline $\begin{array}{c}\text { Times that } \\
\text { this pattern } \\
\text { has } \\
\text { appeared }\end{array}$ & Cascading Events patterns \\
\hline 9744 & NSG2-UV \\
\hline 6046 & NSG-UV, NSG3-UV \\
\hline 399 & NSG-UV \\
\hline 371 & $\begin{array}{c}\text { NSG2-UV, NSG3-UV, NSG1-UV, G2-UV, Load_C- } \\
\text { UF, Load_B-UF, Load_A-UF, G3-US, G1-US }\end{array}$ \\
\hline 349 & $\begin{array}{c}\text { NSG2-UV, NSG3-UV, G2-UV, Load_C-UF, Load_B- } \\
\text { UF, Load_A-UF, G1-US, NSG1-UV, G3-UV }\end{array}$ \\
\hline 335 & $\begin{array}{c}\text { NSG2-UV, NSG3-UV, NSG1-UV, G2-UV, Load_C- } \\
\text { UF, Load_B-UF, Load_A-UF, G1-US, G3-UV }\end{array}$ \\
\hline
\end{tabular}

2) Wind penetration impact: The number of protection devices that trip and the reason for tripping as function of the total wind generation output for $130 \%$ system loading are presented in Fig. 2. The number of tripping protection devices due to under-frequency is higher than due to under-voltage regardless of the wind penetration. However, when the wind penetration exceeds a threshold, it seems that the number of protection relays that trip gets higher. In this case for $130 \%$ system loading, when the wind generation output increases over $90 \%$, it has been noticed that more protection devices trip mainly due to under-frequency, and in a more frequent manner. This can be explained by the disconnection of synchronous generation and the resulting lower inertia that the introduction of RES causes.

In an attempt to understand better the effect that wind penetration has on the appearance of cascading events, an investigation on how many devices trip when there is RES penetration at only one area at a time has been performed. When there is wind generation only at Area 1, no protection devices trip. In Fig. 3. it can be noticed that as the wind penetration at Area 2 and the system loading increase, the number of protection devices that trip gets higher. More specifically, there seems to be a threshold that changes with system loading, which when exceeded causes a significant rise in protection device trippings. For example, for this network and for area 2 this threshold is around $50 \%$ for $130 \%$ loading, $70 \%$ for $120 \%$ loading and almost $100 \%$ for $110 \%$ loading.

On the other hand, in Fig. 4. a very different behaviour is seen, highlighting the complexity of the system dynamic behaviour and the need for systematic characterisation of possible cascading events. When there is wind penetration only at Area 3 no protection devices trip when the system loading is $110 \%$. When the system loading is $120 \%$ there is a trend for protection device trips to reduce as penetration increases, especially above $60 \%$. For system loading $130 \%$ protection device trips are generally high and are not affected by wind penetration in area 3 . Therefore the effect of wind penetration on the appearance of cascading events is difficult to be predicted, it is dependent on the specific network topology and no 'worst-case' scenario can be safely assumed.

The results (for this specific system) suggest that a clear penetration threshold where the possibility of cascading events increases cannot be defined in a straightforward manner, as the dynamic behaviour of the system regarding such events is very complex. Therefore, the resulting rules 
from the suggested framework should be defined in more detail (taking into consideration locational aspects as well as the specific reasons for tripping of devices) rather than as simple thresholds.

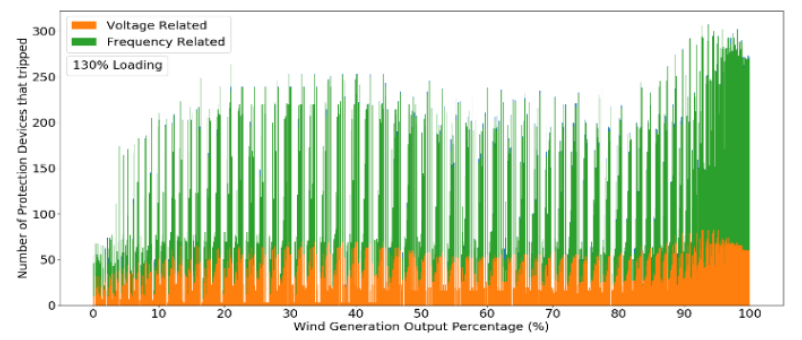

Fig. 2. Number of protection devices that tripped and reason for tripping as function of wind penetration.

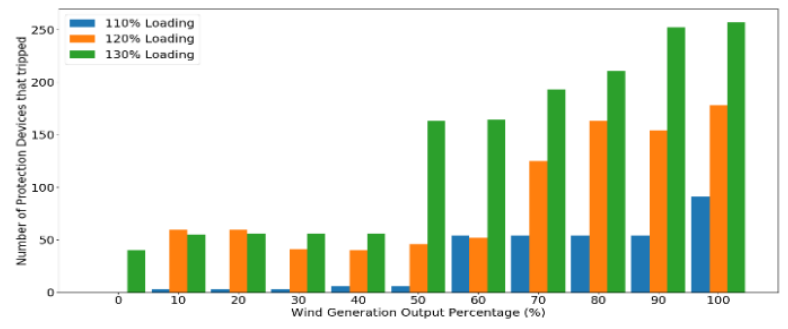

Fig. 3. Number of protection devices that tripped with wind penetration only at Area 2.

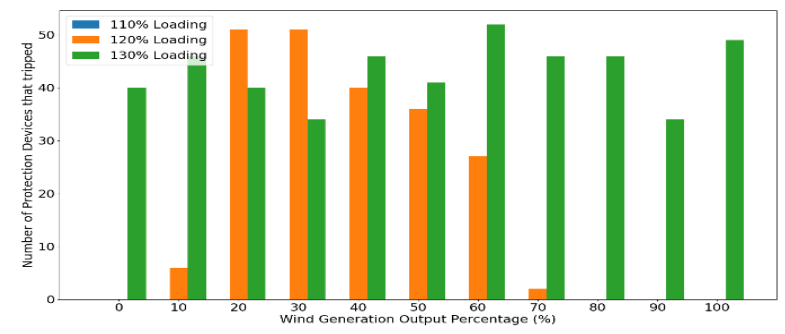

Fig. 4. Number of protection devices that tripped with wind penetration only at Area 3.

3) Locational Aspects, components involved and reasons for tripping: The initating fault location impact on cascading events is presented in Fig. 5. More frequent appearance of cascading events occurs when the fault happens on the lines that are closer to Area 2. In Fig. 6. the number of patterns that each component has appeared in along with the reason for tripping are presented. The synchronous machine G2 gets disconnected in all the cases due to under-voltage and appears in most patterns (156 out of 161), whereas G1 and G3 get disconnected most of the times due to under-speed (145 and 120 out of 161 respectively) and in less patterns due to undervoltage (10 and 24 out of 161). The RES units NSG1, NSG2 and NSG3 get disconnected in all the patterns that they appear in by the UV protection relays, with the NSG2 appearing in higher number of patterns (149 out of 161). It can be concluded that when the initating fault occurs close to Area 2 it has a high impact and that components in that area appear in a high number of cascading events patterns due to disconnection by under-voltage relays.

4) Reason for first cascading event: The first cascading event in 143 out of the 161 patterns is the disconnection of NSG2, in 17 the disconnection of NSG3 and in 1 the disconnection of G2, all due to under-voltage. The time of occurrence of the first cascade and how that is affected by system loading is shown in Fig. 7. The first cascading event occurs earlier, as the system loading increases.

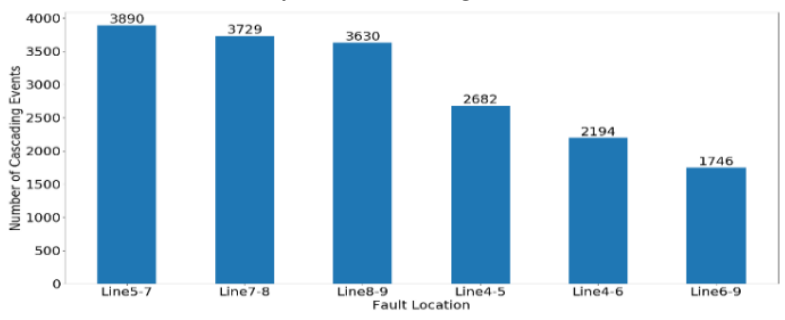

Fig. 5. Number of cascading events according to fault location.

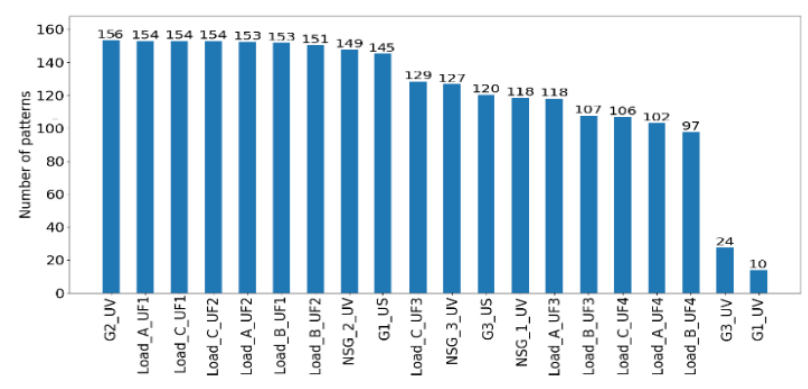

Fig. 6. Number of patterns that each protection device has appeared in.

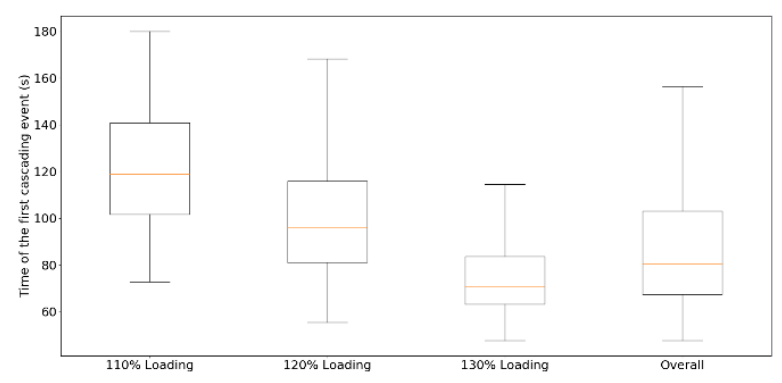

Fig. 7. Time of the first cascading event.

\section{IV.CONCLUSIONS}

In this paper a framework for the characterisation of cascading events in power systems with renewable generation is proposed. It employs long duration time domain dynamic simulations including protection device modelling and investigation of various operating conditions and contingencies to investigate cascading events patterns and how they change. The specific components that trip along with the reason for tripping are analysed. The impact of changes in system loading, wind generation output and synchronous generation disconnection (following an OPF solution) on cascading event patterns is systematically analysed. This information could be vital in understanding network vulnerabilities in terms of weak areas and instability mechanisms and attempt to avoid cascading events from spreading.

The proposed framework is applied on a modified version of the Anderson-Fouad 9 bus model, including RES units. The results highlight the most vulnerable area of the system and the reason for most cascading events. A number of possible cascades for the given network have been identified and characterized and changes in the patterns with system loading and wind penetration have been investigated. The results highlight the complexity of system dynamics as the impact of wind penetration in different areas and at different loading can drastically affect the potential cascades that might appear in 
the system in very different ways. Consequently, any attempt to define thresholds should take into consideration locational aspects as well as the possibility of both deterioration and improvement related to specific mechanisms of cascading events (e.g. voltage or frequency related).

\section{REFERENCES}

[1] J. Bialek, E. Ciapessoni, D. Cirio, E. Cotilla-Sanchez, C. Dent, I. Dobson, P. Henneaux, P. Hines, J. Jardim, S. Miller, M. Panteli, M. Papic, A. Pitto, J. Quiros-Tortos, and D. Wu, "Benchmarking and validation of cascading failure analysis tools," IEEE Transactions on Power Systems, vol. 31, no. 6, pp. 4887-4900, Nov 2016.

[2] IEEE Task Force on Understanding, Prediction, Mitigation and Restoration of Cascading Failures, "Risk assessment of cascading outages: Methodologies and challenges," IEEE Trans. Power Syst., vol. 27, no. 2, pp. 631-641, May 2012.

[3] J. Hazra and A. K. Sinha, "Identification of catastrophic failures in power system using pattern recognition and fuzzy estimation," IEEE Transactions on Power Systems, vol. 24, no. 1, pp. 378-387, 2009.

[4] M. J. Eppstein and P. D. H. Hines, "A "random chemistry" algorithm for identifying collections of multiple contingencies that initiate cascading failure," IEEE Transactions on Power Systems, vol. 27, no. 3, pp. 1698-1705, 2012.

[5] P. Hines, I. Dobson, and P. Rezaei, "Cascading Power Outages Propagate Locally in an Influence Graph that is not the Actual Grid Topology," IEEE Transactions on Power Systems, vol. 32, no. 2, pp. 958-967, 2017.

[6] E. Ciapessoni, D. Cirio, and A. Pitto, "Cascadings in large power systems: Benchmarking static vs. time domain simulation," in Proc. IEEE PES General Meeting, Jul. 27-31, pp. 1-5, 2014.

[7] Weckesser, T., \& Van Cutsem, T. Identifying plausible harmful N-K contingencies: A practical approach based on dynamic simulations. 20th Power Systems Computation Conference, PSCC 2018.

[8] R. I. Hamilton, P. N. Papadopoulos, and K. Bell, "An investigation into spatial and temporal aspects of transient stability in power systems with increasing renewable generation," Int. J. Electr. Power Energy Syst., vol. 115, p. 105486, 2020.

[9] DIgSILENT-PowerFactory User Manual, DIgSILENT GmbH, 2019.

[10] Anderson P, Fouad A, and Happ H. Power system control and stability. IEEE Transactions on Systems, Man, and Cybernetics 1979; 9(2):103103.

[11] Sorensen P, Andresen B, Fortmann J, and Pourbeik P. Modular structure of wind turbine models in IEC 61400-27-1. IEEE Power and Energy Society General Meeting (PES) 2013:1-5.

[12] Dommel $\mathrm{H}$ and Tinney W. Optimal power flow solutions. IEEE Transactions on Power Apparatus and Systems 1968;10:1866-1876.

[13] P. N. Papadopoulos, J. V. Milanović, P. Bhui, and N. Senroy, "Feasibility study of applicability of recurrence quantification analysis for clustering of power system dynamic responses," IEEE PES Innov. Smart Grid Technol. Conf. Eur., 2017.

[14] National Grid Electricity System Operator Limited. (2019). The Grid code | National Grid, (5), 1-998.

[15] Digsilent Technical Documentation. (2013). DIgSILENT PowerFactory Application Guide DPL Tutorial DIgSILENT Technical Documentation. 3-45.

[16] R. Preece and J. V. Milanović, "Efficient Estimation of the Probability of Small-Disturbance Instability of Large Uncertain Power Systems," IEEE Trans.Power Syst., vol. 31, no. 2, pp. 1063-1072, March 2016.

[17] P. N. Papadopoulos and J. V Milanovi, "Efficient Identification of Transient Instability States of Uncertain Power Systems," IREP 2017 Symposium on Bulk Power Systems Dynamics and Control, pp. 1-7, 2017.

[18] IEEE Task Force on Test Systems for Voltage Stability and Security Assessment, "Test systems for voltage stability analysis and security assessment," PES-TR19, Tech. Rep., August 20 